\title{
Mucormycosis in a Patient with Chronic Lymphatic Leukemia in Treatment with Ibrutinib
}

Fodstad $\mathrm{PM}^{1 *}$, Lein $\mathrm{IA}^{2}$ and Tjønnfjord $\mathrm{GE}^{3}$

${ }^{1}$ Department of Ear Nose Throat Surgery, Oslo University

Hospital, Oslo, Norway

${ }^{2}$ Department of Oral Surgery and Oral Medicine, Faculty of Dentistry, University of Oslo, Norway

${ }^{3}$ Department of Hematology, Oslo University Hospital, Oslo, Norway

*Corresponding author: Fodstad PM, Department of Ear Nose Throat Surgery, Oslo University Hospital, P.O. Box 4950, 0424 Nydalen, Oslo, Norway

Received: J une 14, 2021; Accepted: August 04, 2021;

Published: August 11, 2021

\section{Background}

Mucormycosis is a fungal infection caused by fungi belonging to the order Mucorales. The most common pathogenic genera in this order of fungi include Rhizopus and Mucor [1]. The disease was first described by Paltauf in 1885 [2]. The fungi in the Mucoracaeca family are widely found in organic matter such as bread, compost, and animal excrements and exposure is usually by inhalation and direct contact [3]. The most common sites of Mucormycosis infections are the sinuses (39\%), followed by pulmonary (24\%), cutaneous $19 \%)$, cerebral (9\%) and gastrointestinal (7\%). Disseminated disease was seen in $3 \%$ [4].

Mucormycosis is regarded a medical emergency. The treatment is medical combined with surgery if possible. The prognosis varies greatly on the basis of location and extent of the infection as well as the underlying health condition of the patient. Roden et al. reviewed more than 900 cases of Mucormycosis and found an overall mortality rate between $40 \%$ and $80 \%$ [4]. Ghez and colleagues found a mortality rate of 9 out of 32 patients with invasive fungal infections [5].

Chronic Lymphatic Leukemia (CLL) is the most common leukemia in Western countries. It is characterized by accumulation of mature clonal B lymphocytes in blood, bone marrow and secondary lymphoid organs. The clinical course is highly variable. In one third of the patients, the disease is asymptomatic and follows an indolent course not requiring treatment. The remaining patients suffer from active disease with progressive lymphocytosis, bone marrow failure, lymphadenopathy, hepatosplenomegaly and/or B-symptoms and may require several lines of treatment. Autoimmune thrombocytopenia and autoimmune hemolytic anemia are frequent complications of CLL, and they are primarily treated with corticosteroids. Chemoimmunotherapy and more recently treatment with signal pathway inhibitors (ibrutinib and idelalisib) and a BCL-2 inhibitor (venetoclax) have improved survival for CLL patients who require treatment. With the introduction of these novel agents, the patients may experience a new set of adverse effects. An increased frequency of mold infections has been reported in patients treated with ibrutinib, particularly when ibrutinib is combined with corticosteroids [5-7].

\section{Case Presentation}

The patient was diagnosed with CLL in 2010. He was then in his mid-forties. The CLL cells displayed a typical immunophenotype, and they used the IGHV3-53 gene which showed 100\% homology to germ line. FISH-analyses disclosed homozygous del(13q14) and heterozygous del(11q22). In 2015 he received 6 courses of fludarabine, cyclophosphamide and rituximab as third line treatment for CLL. Treatment indications were progressive Binet stage B disease and autoimmune hemolytic anemia in need of high doses of corticosteroids. Complete MRD negative remission was accomplished.

In January 2017 the patient presented with progressive disease (Binet stage B) and autoimmune hemolytic anemia. Prednisolone $(1 \mathrm{mg} / \mathrm{kg})$ was initiated and he received two courses of fludarabine, cyclophosphamide and rituximab with insufficient efficacy. Molecular genetics disclosed four different TP53-mutations and treatment was changed to ibrutinib 420mg daily from February 2017. Corticosteroids were continued. The patient responded favorably to the treatment, and prednisolone was tapered off to a dosage of $20 \mathrm{mg} /$ day as per April 3, 2017.

In April 2017 the patient experienced moderate pain in the left maxillary area and discharge from the left nose. The condition was believed to be acute sinusitis and treatment with antibiotics and prednisolone $40 \mathrm{mg} /$ day was started. This did not reduce the symptoms and Endoscopic Sinus Surgery was performed at his local ENT department after approximately four weeks of symptoms. Microbiological examination revealed a positive culture of Rhizopus Mucor sensitive to amphotericin B. Liposomal amphotericin B in gauze was placed directly in the maxillary sinus and middle meatus, in addition to intravenously administration at $5 \mathrm{mg} / \mathrm{kg} / \mathrm{day}$. The patient was transferred to the Ear Nose Throat Department at Oslo University Hospital for multidisciplinary treatment.

\section{Investigations and treatment}

At the time of admission in our hospital the patient's general condition was good and afebrile. Examination showed crusts in the 

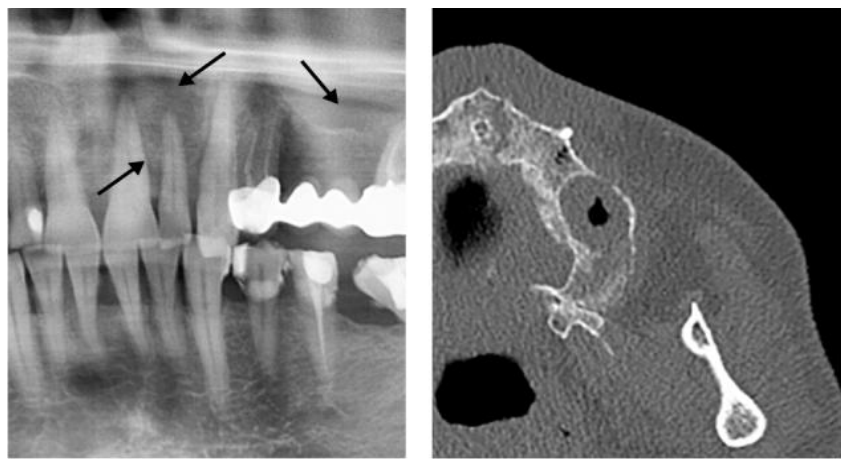

Figure 1: From left A) X-ray of the jaw (Orthopantogram) prior to surgery: Loss of attachment and radiolucent areas around several teeth in the left upper jaw. B) CT of left maxilla demonstrates destruction of bony cortices and general loss of bony details, giving a mottled appearance.
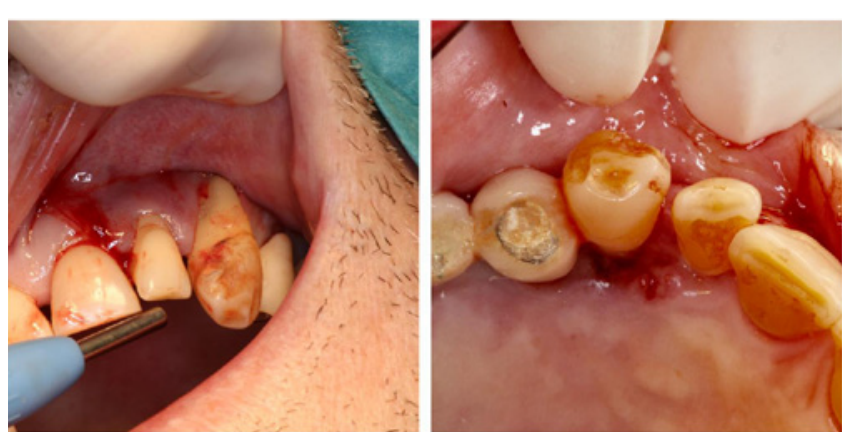

Figure 2: From left A: Picture taken after releasing incision mesial to tooth 22. Findings were gingival retraction and teeth mobility. B) Ulcerated gingiva palatal to 22 and 23 .

left nasal cavity, hypertrophic concha media and numbness of the left side of the upper lip. Mucosal tissue changes were seen in the posterior wall of the maxillary sinus. Computed Tomographic (CT) examination showed signs of sinusitis in the left maxillary, ethmoidal, and frontal sinuses and possible bone erosion in the dorsal maxillary sinus.

The patient was given immunoglobulin $(0.4 \mathrm{~g} / \mathrm{kg}$ i.v. $)$ due to hypogammaglobulinemia $(3.7 \mathrm{~g} / \mathrm{L})$ and a blood transfusion due to anemia $(\mathrm{Hb} 7.8 \mathrm{~g} / \mathrm{dL})$. His CRP was $51 \mathrm{mg} / \mathrm{L}$. Corticosteroids were withdrawn. Ibrutinib was continued at $420 \mathrm{mg}$ daily. Liposomal amphotericin B was increased to $10 \mathrm{mg} / \mathrm{kg}$ due to possible bone involvement.

Radical endoscopic surgery of the left sinuses was performed the day after admittance with wide opening to the maxillary sinus and ethmoids. The posterior maxillary sinus wall was surgically removed to prevent entrapment of fungi in the pterygoid fossa. The CRP continued to climb to $115 \mathrm{mg} / \mathrm{L}$ while the hemoglobin was stable after the intital blood transfer with a hemoglobin around 9g/dL (Figure 1).

Due to progressive loosening of teeth an examination by an oral and maxillofacial surgeon was done. A panoramic X-ray was taken, and the CT was reviewed. Osteolytic lesions around the teeth were disclosed. A bone biopsy was taken with extraction of teeth 22 and 23 . The bone was grey-colored and gave a distinct impression of necrosis and was sent for histological analysis (Figure 2).

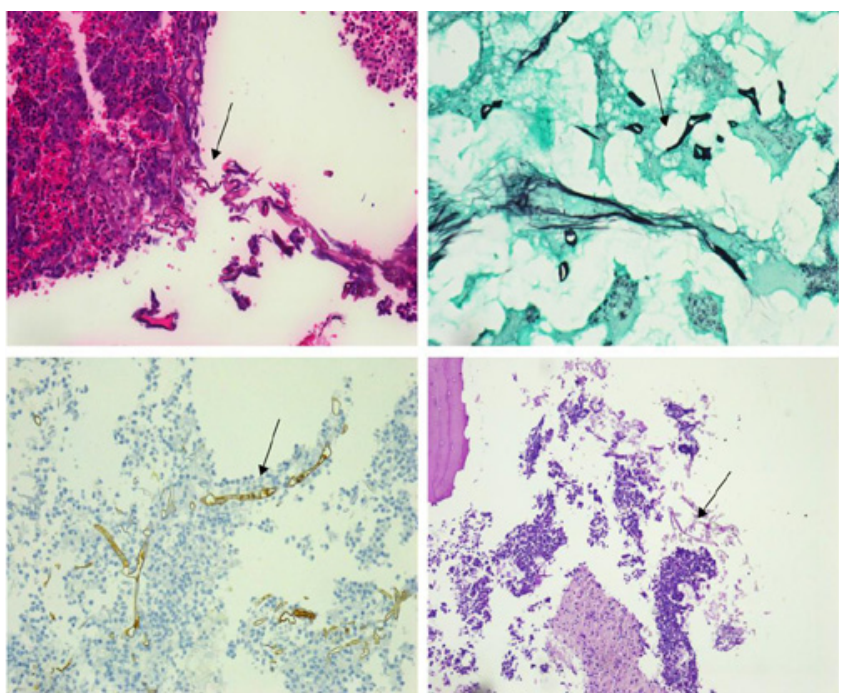

Figure 3: Clockwise from left. Histological appearance from the actual patient. Arrows point to mucor hyphae. A) 200x Hematoxylin and eosin (HE) stain. B) Gomori Methanimine Silver Stain. Hyphae coloured black; C) Gomori Methanimine Silver Stain. Hyphae coloured black; D) Periodic acidSchiff stain (PAS) x 100 .

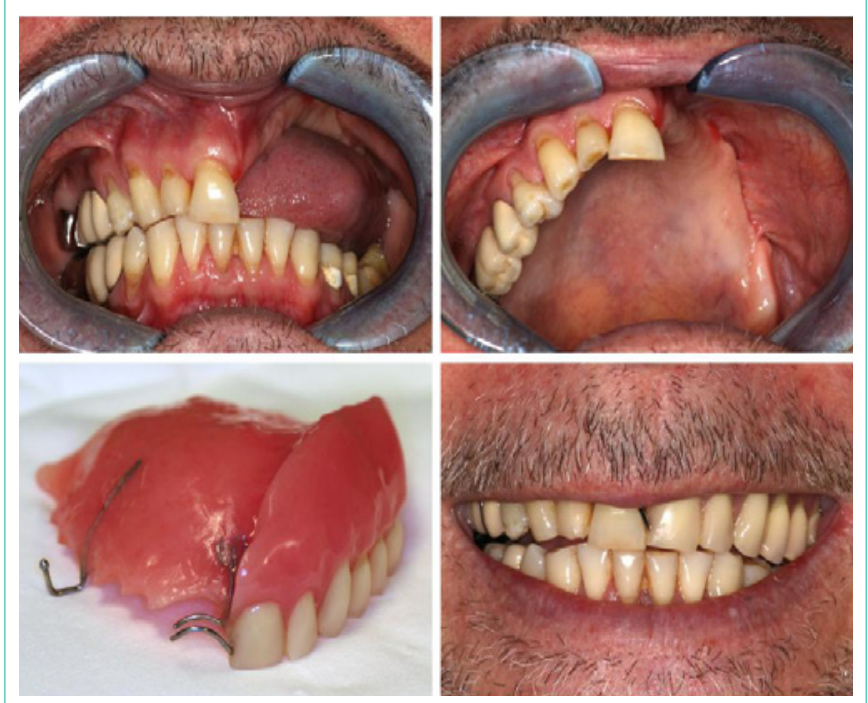

Figure 4: Result after surgery. A dental prosthesis was made with acceptable result.

The strong clinical suspicion of necrosis led to a radical resection with removal of the lower left hemi-maxilla including remaining teeth. The bone specimens confirmed mucormycosis invasion and osteomyelitis. After the surgical interventions the CRP was reduces to levels around 40mg/L (Figure 3).

Repeated endoscopic examination was performed in the coming weeks to verify a disease-free sinus. A dental prosthesis was made with acceptable functional and esthetical result. Two months after the first surgery the CRP was completely normalized below $4 \mathrm{mg} / \mathrm{L}$ (Figure 4).

Six weeks after admission liposomal amphotericin B was substituted by orally administrated posaconazole (300mg once daily) and the patient was discharged. Ibrutinib was discontinued due to 
interactions with posaconazole. Within two months autoimmune hemolytic anemia relapsed, and prednisolone $(1 \mathrm{mg} / \mathrm{kg})$ was resumed. Ibrutinib was restarted although at a low dose; $140 \mathrm{mg}$ every third day. His condition improved continuously, and corticosteroids could be tapered and eventually discontinued. Posaconazole was ceased 12 months after he was diagnosed with Mucormycosis. Ibrutinib was gradually increased in dosage and continued at $420 \mathrm{mg}$ daily. Thereafter the patient enjoyed good health until spring 2019 when he presented with signs of disease progression. Venetoclax (400mg daily) was substituted for ibrutinib with a very favorable response. At present he is considered for an allogeneic stem cell transplant.

\section{Discussion}

This case of mucormycosis demonstrates that subtle symptoms from the orofacial region may represent an invasive pattern of infection. We also show that it may be possible to continue ibrutinib during treatment of an invasive fungal infection.

Mucormycosis and other fungi such as Aspergillus species are readily found in nature and rarely affect healthy individuals. The main risk factors for invasive fungal infections are diabetes, chronic liver disease, treatment with corticosteroids, neutropenia, and bone marrow depleting chemotherapy [5]. In recent years several studies and case reports have shown an increase of Invasive Fungal Infections (IFI) in patients treated with ibrutinib [6,7]. Most often these patients are infected with aspergillus. Mucor genus is rare. Ghez et al found only 1 case of Mucormycosis in a series of 33 cases of IFI in patients treated with ibrutinib [5].

Corticosteroids is a known risk factor for developing fungal infections. Our patient had received several courses with prednisolone previously due to autoimmune hemolytic anemia without adverse side effects. We believe the ibrutinib treatment was the main cause of this patient's mucormycosis since the fungal infection became symptomatic within two months of starting with ibrutinib. We consider the corticosteroids to be a contributing factor. Other authors have found this same quite rapid onset of fungal infections. After ibrutinib treatment $[5,7,8]$.

The mechanism behind ibrutinib associated fungal infections is not fully understood. Several pathways have been proposed. They include macrophage $\mathrm{BtG}$ inhibition that may decrease the granulocyte response to fungal infections [9]. Blood samples from patients treated with ibrutinib show reduced oxidative burst and absent IL-8 secretion in neutrophil granulocytes in in vitro studies with Aspergillus stimulation [10]. Ibrutinib easily penetrated the blood-brain- barrier. Animal studies have shown a fourfold increase of intracerebral concentration of ibrutinib compared to extracranial in mice [11]. If this holds true in vivo it may help explain the high incidence of intracerebral involvement in patients with IFI treated with ibrutinib. Ruchlemer et al. reported CSF involvement in $41 \%$ of their cases [6].

We chose to continue ibrutinib treatment during the treatment period. Most authors favor to stop ibrutinib in the case of a fungal infection. In Ruchlemers report of 35 patients with IFI on ibrutinib treatment, the medication was withdrawn in $91 \%$ of the cases, and the authors recommend doing so [6]. Our reasoning for not stopping ibrutinib was that the patient was in need of treatment due to autoimmune hemolytic anemia secondary to CLL. We considered continuing ibrutinib to be a better choice than high-dose corticosteroids, and ibrutinib has no interaction with amphotericin B. When amphotericin B was substituted with posaconazole, we stopped ibrutinib on the reason that posaconazole increases ibrutinib concentration 5-15 times. Posaconazole is a strong CYP3A4 inhibitor. Ibrutinib treatment was resumed in a reduced dose after a while because the patient was in need of CLL directed treatment and ibrutinib was continued throughout posaconazole treatment with good tolerance.

Medical and surgical treatment was given on the basis of best clinical practice at the time. International Guidelines published in 2019 [1] recommends liposomal amphotericin B 5-10mg/kg per day as first line treatment. Our treatment was in concordance with these later published guidelines. Surgical intervention was performed with the goal of removing all involved tissue without unnecessary mutilation. The reason for such radical surgery is the eroding and invading of blood vessels by the Mucorales species, followed by thrombosis, ischemia and tissue necrosis [12]. This leads to reduced local vascularization and hence reduced local concentration of amphotericin B. In our case part of the maxilla was removed and later a prosthesis was made with satisfying functional and aesthetic result.

\section{Conclusion}

Ibrutinib treatment is a risk factor for invasive fungal infections. Symptoms involving sinuses, teeth and the jaws must be taken seriously, as they can be a sign of invasive infection. Before starting ibrutinib risk factors for invasive fungal infections must be investigated. Medical and if possible, surgical treatment must start without delay. We managed the invasive fungal infection successfully without cessation of ibrutinib which is an option to consider if presented with a case like this.

As of today, no guidelines exist on whether to give prophylactic anti-fungal treatment to patients on ibrutinib or whether to stop ibrutinib in case of a fungal infection. Further investigations into these matters are needed.

\section{Acknowledgement}

The authors would like to thank Dr. Peter Jebsen for help with the pathology section and Mrs. Ine Eriksen for help with the figures.

ICMJE Statement: All authors have contributed to the writing of the manuscript. All authors have followed the patient in a clinical setting and have substantially contributed to the planning, execution and revisions of this manuscript.

\section{References}

1. Cornely OA, et al. Global guideline for the diagnosis and management of mucormycosis: an initiative of the European Confederation of Medical Mycology in cooperation with the Mycoses Study Group Education and Research Consortium. The Lancet Infectious Diseases. 2019; 19: e405-e421.

2. Paltauf A. Mycosis mucorina. Virchows Arch. 1885; 102: 543-564.

3. Lion TE, David. Human Fungal Pathogen Identification and epidemiology. 2017: 37.

4. Roden MM, et al. Epidemiology and outcome of zygomycosis: a review of 929 reported cases. Clin Infect Dis. 2005; 41: 634-653.

5. Ghez D, et al. Early-onset invasive aspergillosis and other fungal infections in 
patients treated with ibrutinib. Blood. 2018; 131: 1955-1959.

6. Ruchlemer R, et al. Ibrutinib-associated invasive fungal diseases in patients with chronic lymphocytic leukaemia and non-Hodgkin lymphoma: An observational study. Mycoses. 2019; 62: 1140-1147.

7. Varughese $\mathrm{T}$, et al. Serious Infections in Patients Receiving Ibrutinib for Treatment of Lymphoid Cancer. Clin Infect Dis. 2018; 67: 687-692.

8. Eichenberger EM, et al. A case of CNS aspergillosis in a patient with chronic lymphocytic leukemia on first-line ibrutinib therapy. Med Mycol Case Rep. 2020; $27: 17-21$
9. Maffei R, et al. Immunomodulatory effect of ibrutinib: Reducing the barrier against fungal infections. Blood Reviews. 2020; 40.

10. Blez D, et al. Ibrutinib induces multiple functional defects in the neutrophil response against Aspergillus fumigatus. Haematologica. 2020; 105: 478-489.

11. Goldwirt L, et al. Ibrutinib brain distribution: a preclinical study. Cancer Chemotherapy and Pharmacology. 2018; 81: 783-789.

12. Metzen D, et al. Mucormycosis of the head and neck. J Craniomaxillofac Surg. 2012; 40: e321-e327. 